\title{
The risks of nanomaterial risk assessment
}

\author{
The addition of carbon nanotubes to the SIN ('Substitute It Now') list has evoked reactions from nanomedicine \\ and nanotoxicology researchers, who ask for a fairer and more recent research-based risk assessment of carbon \\ nanotubes.
}

$\mathrm{T}$ he SIN list (https://chemsec.org/ sin-list/) is developed by the Swedish non-profit organization ChemSec (in collaboration with an advisory board comprising several non-governmental organizations), and contains hazardous chemicals and nanomaterials that ChemSec believes should be restricted or banned in the EU. The list is based on the substances registered as Substances of Very High Concern (SVHC) in REACH (the Registration, Evaluation, Authorisation and Restriction of Chemicals regulation) - the EU regulatory framework for industrial chemicals; however, ChemSec claims to be faster in implementing new hazardous substances by evaluating recent scientific research and by not being slowed down by the EU regulatory machinery, thereby offering a suitable guideline for companies, regulators and authorities to ensure non-toxic and safe products (S. F. Hansen and A. Lennquist, Nat. Nanotechnol. 15, 3-4; 2020).

When it comes to nanomaterials, risk and toxicity assessments are challenging tasks, because biological and pathological effects are determined by a variety of parameters, such as size, shape, basic chemical structure, charge and protein corona. In fact, size alone might not matter as much when it comes to toxicity and biopersistence. In addition to material properties, exposure route and dosage need to be considered to properly analyse potential risks to the human body and to define the appropriate safety measures. Evaluating these specific biological interactions is a major subject of ongoing research in the field of nanomaterials, and indeed, they might only be assessable on a case-by-case basis, that is, each modification might change the biological fate.

Two Correspondences in this issue, one by Bengt Fadeel and Kostas Kostarelos, and one by Daniel A. Heller and colleagues, highlight the problems and challenges specific to carbon nanotube risk assessment. In these pieces, nanomedicine and nanotoxicology researchers demand that carbon nanotubes should not be viewed as one type of material, as advocated by ChemSec, but that they should be grouped by their individual features, dosing and exposure routes. To achieve a fair risk assessment of carbon nanotubes, both Correspondences recommend a detailed evaluation of recent insights into the shortand long-term interactions of the different types of carbon nanotubes in the correct in vivo models and the potential adverse effects in the context of the applications for which they were designed for.

The basis of effective nanomaterial risk assessment is a high standard of characterization and reporting (including the reporting of negative results). To which degree and how such reporting standardization can be achieved is an ongoing discussion in the field (M. Faria, et al. Nat. Nanotechnol. 13, 777-785; 2018) and will require a more concerted effort from the scientific communities. In turn, this information will provide the correct basis for the assessment of potential toxicities by regulatory agencies, frameworks and non-profit organizations.

Published online: 10 March 2020

https://doi.org/10.1038/s41565-020-0658-9 